\title{
Une brève histoire de la notion de qualité des aliments en France: du consensus aux controverses
}

\author{
Carole Chazoule, Université de Lyon \\ Rémy Lambert, Université Laval ${ }^{1}$
}

\section{Introduction}

Depuis que les économistes des conventions ont ouvert la boîte noire de la qualité, une nouvelle conception de la notion a émergé et s'est peu à peu imposée. On peut rapidement la résumer en quelques phrases: la qualité est une construction sociale, intimement liée aux évolutions industrielles, aux mouvements économiques et à l'histoire des sociétés. La notion qui est polysémique arbitraire, relative, mouvante en fonction des périodes et des institutions n'est pas exempte de subjectivité ${ }^{2}$. Elle est donc difficile à définir, surtout si l'on veut lui donner un sens qui s'imposerait à tous au-delà des mouvements socio-économiques qui la construisent. Son analyse, néanmoins, devient possible si l'on s'intéresse à son processus de construction et de qualification. En tant que processus, elle est le résultat d'un arbitrage entre multiples dimensions et multiples acteurs. Cet arbitrage est relatif en ce sens qu'il peut être différent d'une période de l'histoire à une autre, mais aboutit à un consensus social sur "une généralité de la qualité » où le plus grand nombre a alors une conception homogène de ce qu'est la qualité d'un bien ${ }^{3}$.

Dans le secteur agroalimentaire français de ces cinquante dernières années, s'il ne fallait retenir qu'une chose, c'est bien que l'évolution de la perception de la qualité par les différents opérateurs économiques du système agroalimentaire (producteurs, metteurs en marché, transformateurs, distributeurs et consommateurs) a été totalement liée aux évolutions du secteur et de son industrialisation. On peut distinguer trois périodes au cours desquelles un premier consensus s'est formé d'une façon assez implicite puis a évolué au cours des deux secondes vers de plus en plus de codifications et de certifications. Ce premier consensus prend forme après guerre autour de l'accord que la qualité d'un produit agroalimentaire est liée à sa qualité sanitaire. Dans les deux secondes périodes qui couvrent, pour la première, la décennie des années 1980 et pour la seconde, celle des années 1990, la notion de qualité est peu à peu élargie, elle quitte le seul domaine sanitaire pour englober de multiples réalités. De nouveaux critères entrent alors en ligne de compte dans la fabrication des produits agricoles et agroalimentaires pour définir la qualité et segmenter les marchés. C'est à ce moment qu'apparaît une différence entre une qualité de base dite générique, qui s'appuie essentiellement sur des garanties de salubrité, et une qualité dite spécifique, définie par sa capacité à se démarquer d'un produit standard. Cette capacité peut intégrer des considérations nutritionnelles et organoleptiques (plus de variété, plus de goût, plus de vitamines), ou éthiques (comme le respect de l'environnement et du bien-être animal) ou encore culturelles (terroirs, typicités, traditions). L'objectif de cet article est donc de montrer comment s'est fait ce basculement d'une qualité essentiellement définie par des critères sanitaires à une qualité différentielle définie cette fois par des critères variés. Pour cela, dans chacune des périodes que nous avons définies, nous ferons dialoguer le modèle que nous appellerons « productif industriel » et ses évolutions avec la notion de qualité.

\section{0-1980 : une qualité générique réelle, mais non certifiée}

À la fin de la Seconde Guerre mondiale, la France a besoin d'être reconstruite, l'agriculture aussi. Avec enthousiasme, la jeunesse agricole répond au défi qui 
lui est lancé par l'État et les professions agricoles : moderniser le secteur et atteindre l'abondance alimentaire. L'industrialisation permet à la France, en quelques années à peine, de sortir de la situation de pénurie dans laquelle elle se trouvait jusque-là. L'agriculture devient plus technique et plus scientifique sous l'impulsion des cogestionnaires de la modernisation et d'organismes de recherche nouvellement créés tels que l'INRA. L'objectif est d'augmenter la productivité, espérant ainsi abaisser le prix des produits. Les engrais et les pesticides sont mis à la disposition des agriculteurs qui bénéficient également d'une amélioration constante des races et des végétaux. La formation s'accroît, et de nombreux techniciens servent de relais dans les campagnes. Ils diffusent des modes de production et des techniques standardisées permettant l'intensification de la production et la spécialisation des exploitations. Le métier et les exploitations se transforment : c'est la fin du système de polycultureélevage. Le nombre d'exploitations diminue et leur superficie augmente; elles sont maintenant spécialisées et emploient relativement moins de maind'œuvre, les investissements sont facilités. Les résultats ne se font pas attendre, tout autant qu'ils sont soutenus par la Politique agricole commune (PAC). Ils se matérialisent par une augmentation régulière de la productivité agricole, laquelle s'accompagne d'une baisse importante des coûts de production et du prix des produits, d'une part, et d'une croissance des marchés de masse, d'autre part. Avec les bassins de production, les techniques se standardisent de même que les produits. L'ancienne diversité des produits agricoles laisse alors la place à la suprématie de quelquesuns qui, parce qu'ils répondent mieux à la productivité et à la demande de l'époque, s'imposent sur les marchés. Par contre, l'industrie agroalimentaire, bénéficiant d'une amélioration constante de ces techniques, va proposer des produits transformés de plus en plus diversifiés et améliorés. Le transport va se développer et la distribution, s'industrialiser. Des produits jusqu'alors saisonniers et régionalisés vont dès lors se consommer toute l'année et sur tout le territoire. La compétitivité accrue du secteur va rapidement permettre au pays de passer d'un statut d'importateur à celui d'exportateur.

C'est à cette époque que les consommateurs français vont découvrir qu'ils ont à leur disposition des produits sains et propres à la consommation. La qualité de ces produits est définie par des textes réglementaires établissant les niveaux de garantie de sécurité sanitaire ${ }^{4}$. Ces textes, entrés en fonction depuis le début du siècle (où la première loi a été votée), vont se développer à partir des années 1960 et évoluer, pour peu à peu satisfaire aux exigences accrues de sécurité liées aux processus d'industrialisation et à l'augmentation des échanges internationaux. En 1962, la France adopte le Codex Alimentarius puis, en 1971, est créé le service vétérinaire d'hygiène alimentaire qui pose les bases générales de la sécurité sanitaire (cf. Encadré 1). Les contrôles s'effectuent autant dans les exploitations qu'à l'intérieur des usines de transformation où l'innovation se développe autour de nouveaux processus de fabrication des produits. Dans la majorité des cas, ce sont les entreprises elles-mêmes qui assurent les contrôles, mettant en jeu leur réputation en cas de problèmes. La sécurité sanitaire est de la même façon de plus en plus sauvegardée par une amélioration des conditions de conservation et de transport. Cette qualité, par contre, à l'exception du « label rouge », n'est pas ou très peu labellisée dans les produits finaux. Ainsi, les consommateurs désireux de la vérifier se trouvent devant de vraies difficultés, car l'information manque. Leurs actes d'achat se fondent donc uniquement sur la confiance qu'ils accordent implicitement à leur système agroalimentaire. Confiance renforcée par les industriels qui développent les grandes marques agroalimentaires que nous connaissons aujourd'hui et les grandes et moyennes surfaces qui les mettent à disposition, tout en basant leur stratégie marketing sur une politique de qualité à petit prix en créant leurs propres marques. Tout est fait pour assurer la confiance des consommateurs puisque l'homogénéisation de la production leur permet de retrouver, d'un achat sur l'autre, des produits identiques et facilement identifiables.

\section{En 1962, la France adopte le Codex Alimentarius puis, en 1971, est créé le service vétérinaire d'hygiène alimentaire qui pose les bases générales de la sécurité sanitaire.}

Ainsi définie, la notion de qualité fait rapidement l'objet d'un consensus social qui relève du contrat moral. Ce consensus prend forme parce qu'effectivement, la qualité s'améliore sur de nombreux points. Pour la première fois, la menace de nombreuses maladies liées à des carences alimentaires s'éloigne ${ }^{5}$. Quant aux décès et maladies graves encore imputables 
à l'alimentation, ils proviennent plus souvent des comportements ou des choix des consommateurs euxmêmes que de la sécurité des produits. Les consommateurs manifestent alors une grande confiance dans le système en place et la qualité de leur alimentation, sans que ne s'exprime aucun besoin de certification pour la justifier. Il faut dire cependant que le marché, alors en expansion, ne nécessite pas de développer une politique de qualité basée sur une différenciation et les rentes que celle-ci peut amener. Ni les agriculteurs ni la distribution qui est en train de s'industrialiser n'en ont l'utilité pour atteindre leur niveau d'efficacité économique. Cela explique sans doute pourquoi les signes officiels de qualité (cf. Encadré 2) dont les dispositifs juridiques et institutionnels sont en place depuis le début du siècle restent encore confidentiels, répondant deçà delà à un besoin de développement agricole et de compensation pour les régions désavantagées par rapport aux conditions d'implantation de l'agriculture intensive. Les AOC ont été créées en premier. Elles se sont construites sur les notions de délimitation (loi 1905) et de terroirs (loi 1919) afin de lutter contre une concurrence déloyale dans la viticulture. La loi de 1996 définit l'AOC comme « la dénomination géographique d'un pays, d'une région ou d'une localité servant à désigner un produit qui en est originaire et dont la qualité et les caractères sont dus exclusivement ou essentiellement au milieu géographique comprenant les facteurs naturels et humains ».

\section{En quelques années, la confiance établie précédemment dans le modèle industriel est remise en cause.}

\section{Les années 1980 : premières remises en cause}

La réussite du modèle industriel est flagrante jusque dans le milieu des années 1980 où, en quelque sorte victime de son succès, la modernisation de l'agriculture ne va plus apparaître sans conséquence. Celles-ci vont se révéler peu à peu tout au long de cette fin de siècle et vont s'exprimer tant sur le plan social, économique qu'environnemental. En quelques années, la confiance établie précédemment dans le modèle industriel est remise en cause. Tout commence avec l'apparition des premières grandes crises économiques et de surproduction. Malgré la baisse des prix des produits, la croissance de la consommation n'est pas au rendez-vous. L'ouverture des marchés n'absorbe pas l'excédent, elle renforce au contraire la concurrence. Rapidement, le marché et la consommation atteignent des taux de saturation record et les premières crises excédentaires apparaissent. Les producteurs voient leurs rémunérations diminuer et ce, d'autant plus qu'avec la modernisation de l'agriculture, la plupart d'entre eux ont délégué l'essentiel de leur compétence de commercialisation à des circuits élargis nationaux et internationaux ${ }^{6}$. À tout cela s'ajoutent des tensions internes très fortes sur la répartition des moyens de production aussi bien entre producteurs qu'entre régions (concentration ici, désertification là), avec partout une accélération de l'exode rural. Malgré l'importance de ces crises, leur gestion politique reste partielle durant toute la décennie 1980, et aucune réelle remise en cause n'est observée. Attribuant les crises à une mauvaise gestion des excédents par la PAC, la politique agricole soutient qu'elles sont plus conjoncturelles que structurelles et met en place des mesures dont le rôle est de limiter la production (ou sa mise en marché). Les résultats des premiers débats engagés dans les années 1980 par Édith Cresson, alors ministre de l'Agriculture, en témoignent. Ceux-ci n'abordent non seulement pas encore les questions de l'environnement ou de la qualité des produits, mais surtout n'ont pas pour objectif d'aboutir à une réorientation du système productif agricole. Motivés par la seule crainte d'un effondrement de l'emploi dû à un accroissement important de la productivité, ils débouchent sur l'instauration des quotas laitiers, des mesures de mise en jachère ou encore de retraits. Dès lors, l'élimination des excédents par la communauté se généralise. De grandes quantités de lait et de beurre sont détruites. Le coût de soutien public alloué à l'agriculture ne cesse d'augmenter alors même que les budgets nécessitent une gestion au plus juste. Le soutien procuré par la PAC est «coûteux, sans limites, inégalitaire » et a peu d'impact sur les revenus des producteurs ${ }^{7}$. En même temps, les manifestations d'agriculteurs se développent, on les voit aux frontières tenter d'enrayer l'importation. Ils témoignent d'un réel malaise, celui-là d'autant plus important qu'on accuse les producteurs de la destruction de l'environnement, de la pollution des eaux et de celle de la terre. Certains auteurs, pour qualifier ce malaise tant il est fort, parlent de ruptures ${ }^{8}$. Ces ruptures sont de plusieurs ordres à la fois. Les changements opérés depuis l'après-guerre sont si importants et si rapides qu'ils ont modifié la relation de l'agriculteur à son image et 
à son métier, bref à ses anciens savoirs et valeurs. C'est une vraie révolution par laquelle est passée l'agriculture, une révolution telle que celle qui avait marqué l'étape du passage de chasseur cueilleur à celui de cultivateur. Dans cette révolution, l'agriculteur est devenu un producteur parmi d'autres. Il a perdu son statut particulier de «nourrisseur» pour devenir un producteur, chef d'entreprise. Quant à sa production, elle n'est plus essentiellement que celle de matières premières recyclées dans les circuits de l'industrie agroalimentaire. Une distance s'est définitivement installée entre l'agriculteur et le consommateur pour qui la production est le fruit d'une vraie «boîte noire ». Il est incapable de dire comment les aliments qu'il consomme sont produits. Quant à la terre, les animaux d'élevage, les espèces végétales, ils sont devenus des outils de production qu'on ne cesse d'améliorer afin d'en augmenter la productivité. Avec l'apparition des grands bassins de production, l'amélioration des techniques et la délocalisation des cultures dues notamment au développement du hors sol, la disparition de la diversité régionale ainsi que la « saisonnalité " des produits, l'origine et les caractéristiques propres à chaque produit alimentaire sont de plus en plus indifférenciées, voire niées.

Ces ruptures et les crises qui les accompagnent ne sont pas sans conséquence sur la perception de la qualité que se font les consommateurs qui, pour la première fois, s'interrogent. Ils ne savent plus comment les produits sont fabriqués, ni ce qui se cache derrière les frontons des grandes entreprises agroalimentaires et demandent des comptes. Au fur et à mesure de l'industrialisation du système agroalimentaire, les produits se sont majoritairement coupés de leurs origines et de leurs traditions de production, tant et si bien que, dans les années 1980, les consommateurs sont tout autant éloignés des processus de production et de transformation que les produits le sont majoritairement de leur région d'origine. Aussi il n'est pas étonnant que les remises en cause du système productiviste viennent fortement ébranler la confiance des consommateurs, elle qui ne reposait sur rien de codifié ni de vérifiable pour eux. Et il n'est pas étonnant non plus qu'alors, une demande de renégociation de l'ancien contrat social se fasse sentir. En second lieu, c'est par une remise en question, d'une part, de la qualité organoleptique et nutritionnelle des produits et, d'autre part, de l'activité agricole quant à ses conséquences sur l'environnement et la santé que les consommateurs interrogent leur ancien contrat social sur la qualité. Les critiques faites au système industriel concernant ses conséquences sur l'environnement s'intensifient alors, distillant auprès des consommateurs un réel sentiment de méfiance. Si bien qu'à la fin des années 1980, la protection de l'environnement devient un véritable enjeu commercial et favorise le développement d'îlots d'agriculture dite «plus saine » et, en tous les cas, "moins polluante ». C'est le développement de l'agriculture biologique. De plus en plus d'agriculteurs se convertissent à ce type de production au fur et à mesure que le marché se développe, puis des réseaux de distribution émergent avec l'apparition de petites coopératives de vente. Ce mouvement va de pair avec une certaine lassitude qui s'installe par le manque de diversification des produits agricoles proposés sur les marchés. On assiste alors peu à peu au développement des produits sous signes officiels, et notamment les labels et AOC qui, vers la fin des années 1980, sortent de leur confidentialité.

\section{Dans cette révolution, l'agriculteur est devenu un producteur parmi d'autres. Il a perdu son statut particulier de « nourrisseur » pour devenir un producteur, chef d'entreprise.}

\section{Les années 1990 : la fin d'un modèle unique}

Lorsque, dans les années 1990, la croissance des échanges sur les marchés mondiaux s'accélère, amplifiant d'autant la compétitivité et la concurrence entre les producteurs, le terreau du renouvellement est en place. Dans le contexte de crise économique qui est le sien au début de la décennie, le modèle classique des «Trente Glorieuses » reposant "sur un cercle vertueux de progrès technique, d'augmentation de la productivité, de la consommation et des revenus », fonctionne de moins en moins ${ }^{9}$, et la plupart des certitudes concernant sa domination et sa durabilité sont ébranlées. Les remises en cause du modèle sont telles qu'elles vont trouver pour la première fois un écho dans les réformes de la PAC. La politique agricole commune va en effet opérer des changements témoignant d'une prise en compte du malaise agricole. Elle commence à envisager la place d'une agriculture alternative et durable au sein de la société. En 1992, la PAC connaît une première réforme qui en témoigne ${ }^{10}$. Du côté des consommateurs, la perte de confiance va continuer de s'accentuer peu à peu en se 
renforçant au plus fort des crises sanitaires que connaît la période. Les difficultés rencontrées pour régler d'abord la propagation de 1'ESB (Encéphalite spongiforme bovine ou maladie de la vache folle) puis de la fièvre aphteuse et de la dioxine, de la listéria, de la fièvre porcine et plus récemment de la fièvre aviaire vont ainsi finir de mettre les problèmes environnementaux et de santé publique en plein cœur du débat. Les problèmes, déjà discutés et mis en avant depuis presque dix ans, commencent à toucher un nombre de plus en plus important de consommateurs. Les images de cheptel abattu et brûlé pour éviter la contamination, les agriculteurs au bord de la faillite ont en effet marqué symboliquement et pour longtemps de nombreux esprits. Le mode de production dominant et/ou dit conventionnel est maintenant perçu à de nombreux points comme une menace, et sa durabilité est largement remise en question. Nombreux sont ceux qui se demandent comment le mode de production le plus connu pourra encore se maintenir sans se transformer face aux nouvelles exigences des consommateurs et du marché. Il semble maintenant évident que les relations de l'ensemble du secteur agroalimentaire sont en pleine mutation. Le modèle homogène est en train d'éclater, mais les conditions de naissance d'un autre, fondé sur une légitimité renouvelée du secteur, ne sont pas encore réunies. On peut imaginer néanmoins plusieurs scénarios possibles, allant du prolongement du modèle intensif avec plus ou moins l'introduction de considérations environnementales plus contraignantes, à une rupture très marquée dans la conduite des itinéraires techniques et/ou dans l'organisation des exploitations agricoles ${ }^{11}$. Certains préconisent que seule une continuité industrielle permettrait une régularisation du marché; pour les autres, seule une rupture radicale autoriserait l'avènement d'un modèle agricole en phase avec les attentes d'une partie des consommateurs, la protection sanitaire et environnementale. Dans un cas comme dans l'autre, la réponse ne semble pas satisfaire complètement les consommateurs. Entre ces deux possibilités radicales, d'autres possibilités se dessinent. Lacombe (1998) et Roger (2001) envisagent déjà la co-existence de plusieurs modèles parallèles définissant l'agriculture future. Mais plus à la croisée des chemins, c'est l'agriculture durable, intégrée, multifonctionnelle ${ }^{12}$ (selon les termes utilisés) qui semble offrir, pour un temps du moins, une voie d'avenir sans pour autant renier le système industriel dans son ensemble ${ }^{13}$. Ici, l'organisation du système industriel est réaménagée, mais il continue d'exister.
Ainsi donc, la prophétie de la fin de l'agriculture productiviste n'aura pas autant d'audiences que celle de la fin des paysans. Sa substitution par un modèle plus écologique ne s'est pas encore accomplie, et il est à ce jour difficilement concevable qu'elle soit facilement remplacée. Sa réversibilité est jugée coûteuse, illusoire, voire impossible, souligne Roger (2001) sans remettre en cause la compétitivité de l'agriculture et l'équilibre économique des exploitations.

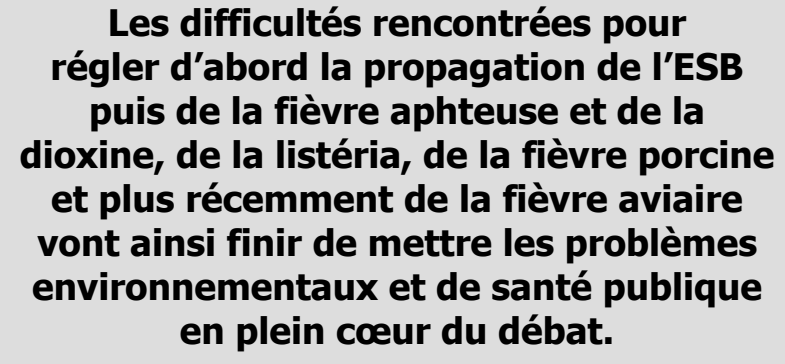

Quant à la question de la qualité, la crise de confiance est maintenant installée, elle prend corps dans ce qui concerne à la fois l'hygiène et la sécurité alimentaire et le nouveau besoin de qualité spécifique des produits alimentaires. Après la crise de l'ESB, les consommateurs commencent à délaisser les filières traditionnelles pour se tourner vers celles de qualité certifiée. Le besoin de réassurance quant à la salubrité, d'une part, et de reprise de contact, d'autre part, avec le produit, son origine, son mode de fabrication vient alors réorienter complètement l'idée de qualité ${ }^{14}$. C'est à ce moment qu'on voit se développer les méthodes portant sur les procédures de production (agriculture raisonnée, normes ISO, HACCP et traçabilité) certifiant la salubrité du processus de production et du produit (cf. Encadré 1). Dans certaines filières, les interventions seront plutôt d'origine publique (réglementations publiques dans les filières viandes) et, dans d'autres (filières végétales), elles vont surtout avoir comme origine des opérateurs privés (et notamment ceux de la grande distribution nord-européenne. C'est aussi à ce moment que l'on voit exploser les filières de qualité spécifique, là encore issues de certifications publiques ou privées. L'exemple des filières « viande » est typique. Alors que la consommation de bœuf et de viandes issues des filières traditionnelles va connaître de fortes périodes de baisse signifiant la méfiance des consommateurs envers des produits dont les informations sur la provenance ou le mode de production sont absentes, on voit se multiplier les applications 
HACCP, les filières de traçabilité, mais aussi se populariser les filières de Certification conformité produit (CCP) ${ }^{15}$ et les labels rouges. Quant aux boucheries traditionnelles, elles connaissent un nouvel essor. Les filières viandes ne sont pas les seules à avoir développé des stratégies de différenciation par la qualité à la suite des grandes crises des années 1990. Les AOC vont sortir de leurs productions privilégiées que sont le vin et les fromages pour s'étendre aux fruits, aux légumes, aux viandes... Les labels vont connaître la même progression qui est encore plus marquée depuis le développement des signes européens et de l'IGP (cf. Encadré 2). Dans le même temps, les marques industrielles, les marques privées de produits (de plus en plus associées à des stratégies de club) ${ }^{16}$ et les marques de distributeurs ou de filières (qui développent leurs propres cahiers des charges ou non) connaissent auprès des consommateurs un réel succès ${ }^{17}$. Ces dernières développent un marketing qui tour à tour évoque l'aspect terroir, tradition et/ou organoleptique des produits, garantissent la traçabilité, jouent sur la mise en place de jurys de consommateurs élisant les produits de l'année ou encore jouent une refonte des rapports de force (concurrence monopolistique) au sein des filières. Ces stratégies de différenciation impliquent de fournir à une clientèle de masse des produits de plus en plus individualisés, mais à coût réduit. Il s'agit de dépasser le dilemme classique productivité/qualité, en alliant, dans une économie de variété, clientélisation et grande série ${ }^{18}$. À partir des années 2000, elles se développent tout autant dans le secteur des produits transformés que dans celui des produits frais. Parfois exclusivement privées, parfois utilisant des certifications publiques (notamment la CCP), ces stratégies se basent sur des accords souvent exclusifs avec des producteurs. Les unes et les autres connaissent également un réel succès qui les positionne aujourd'hui en concurrence directe avec les signes de qualité. Cependant, les signes officiels sont les seuls à proposer une réelle réassurance de la qualité et de la sécurité des produits aux consommateurs, leurs cahiers de charges étant les seuls à être certifiés et contrôlés par des organismes tiers indépendants. À la fin de notre siècle, on ne peut nier que le contrat social entre les différents opérateurs du système agroalimentaire soit en pleine renégociation. La demande des consommateurs a bien été entendue à la fois par les pouvoirs publics et par les opérateurs économiques du système. Et ce, pour les pouvoirs publics qui ont développé des procédures de certification et pour les entreprises pour lesquelles les stratégies de diver- sification des produits et de segmentation des marchés sont devenues un des objectifs dominant de valorisation de leur production dans leur univers de plus en plus concurrentiel et mondialisé.

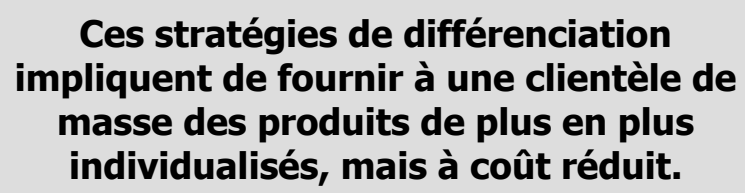

\section{Conclusion}

L'agriculture française est plurielle, elle sort d'un modèle unique, ce qui ouvre de nombreux débats et de nouvelles perspectives en termes de qualité. D'abord essentiellement générique, fruit d'un modèle de développement industriel, voilà la qualité devenue également spécifique avec la remise en question de la domination de ce même modèle. Particuliers et donc multiples, les objectifs de cette qualité spécifique sont tout à la fois des objectifs de sécurisation et de diversification de la production, de segmentation des marchés, de promotion des revenus agricoles, de protection de l'environnement et d'aménagement du territoire. Elle est prise en charge par les pouvoirs publics dans le cas des signes officiels de qualité, et par des acteurs privés souvent innovateurs dans le cas des marques (privées ou collectives, d'entreprises ou de produits) et du développement d'organisations de type Club. Le nombre des signaux de qualité est aujourd'hui important, tout comme le nombre de produits certifiés qui s'est également beaucoup accru ces dernières années sur le marché français. Le phénomène n'est pas sans conséquences et pose de nombreuses questions, notamment en termes de régulation de la concurrence et de perception des différents signaux officiels et non officiels par les consommateurs. Cette pluralité peut-elle trouver toute sa place sur les marchés nationaux, européens, voire mondiaux ? La multiplication des ces signaux certifiés ou non, le manque d'homogénéité entre eux, les différents objectifs qu'ils poursuivent en termes de qualité ne risquent-ils pas, à long terme, de poser des problèmes de lisibilité auprès de consommateurs souvent insuffisamment informés ? La question est d'actualité alors que la politique européenne a validé depuis quelques années des signes officiels européens d'identification de la qualité. 
Au Québec et au Canada en général, les crises ont été moins ressenties, mais des questions se posent tout de même de plus en plus. Ainsi, à l'instar des consommateurs français, de plus en plus de consommateurs québécois s'interrogent sur la qualité des aliments qu'ils consomment. Dans ce contexte de mutations, l'évolution et la définition de ce concept soulèvent de nombreuses interrogations. Pour tenter d'y répondre, et pour donner un guide aux divers intervenants qui auront à rassurer la société québécoise quant à la qualité des aliments qu'elle consomme, par exemple par l'adoption des appellations d'origine contrôlée, cet article s'est proposé, sous un angle quelque peu historique, d'être un voyage au cœur de la notion.

\section{Encadré 1}

1905: Le code rural et celui de la consommation sont créés avec la première loi française en matière de répression des fraudes et de falsification des produits agricoles et alimentaires.

1962: Le Codex Alimentarius des Nations Unies établit des normes volontaires et internationales en matière d'aliments.

1979: Un nouvel accord international est signé dans le cadre du GATT; il donnera lieu à l'Organisation mondiale du commerce.

1984 : Réactualisation du code de la consommation obligeant à indiquer la raison sociale du fabriquant, son nom, son adresse et celle de l'emballeur. L'étiquetage de denrées alimentaires devient obligatoire en France.

Fin des années 1980 : Arrivée de l'ISO 9000 dans l'agroalimentaire.

1993 : Directive hygiène 93/43 qui impose l'application de démarche de type HACCP et principe de traçabilité.

1995 : Création du Conseil national de l'alimentation (CNA).

1997: Système d'identification, d'enregistrement et d'étiquetage obligatoire de la viande bovine (origine, catégorie et race de l'animal). Début de la révision du Codex Alimentarius touchant les biotechnologies et l'innocuité des aliments.

1998: Obligation pour les professionnels français de l'agroalimentaire de s'autocontrôler selon la méthode d'analyse du risque HACCP (Hazard analysis critical control point). L'étiquetage des produits transformés contenant des OGM est rendu obligatoire en Europe.

1999: Création de l'AFSSA (Agence française de sécurité sanitaire des aliments) chargée de l'évaluation des risques sanitaires. La même année, la loi française impose aux acteurs de la chaîne de distribution des procédures permettant la traçabilité des produits.

2000 : Livre blanc de la Commission européenne concernant la stratégie globale de sécurité sanitaire dans l'Union Européenne. Tous les produits contenant plus de $1 \%$ d'OGM doivent être étiquetés, les farines carnées sont interdites dans l'alimentation de tous les animaux d'élevage.

Aujourd'hui, au niveau européen, la directive 2002R0178 du $1^{\mathrm{er}}$ octobre 2003, appelée « Food low », remplace la directive 93/43 et impose de nouvelles exigences européennes en termes de traçabilité. À la suite de ce texte général, des directives européennes sectorielles verront le jour courant 2004. Certains arrêtés seront donc certainement modifiés au niveau français. Cette directive rend obligatoire la déclaration des établissements qui fabriquent des denrées végétales, ce qui n'était pas le cas avant.

\section{Encadré 2}

Aujourd'hui, en France, on recense plusieurs signes d'identification de la qualité et de l'origine (SIQO) définis par la loi d'orientation agricole de 1999 : les SIQO sont les AOC (appellation d'origine contrôlée), la labellisation « Montagne » qui certifie une origine, les labels agricoles (aussi dits label rouge), la certification de conformité produit (CCP) qui certifie une qualité supérieure, la certification biologique $(\mathrm{AB})$ qui certifie un mode de production respectueux de l'environnement et les protections européennes (Indication géographique protégée IGP, Appellation d'origine protégée (AOP) et Attestation de spécificité (AS). (Rapport du CNA 2003.) 


\section{Notes et références}

1 Respectivement enseignante-chercheure, ISARA-IER, Université Lyon et professeur titulaire chercheur, Centre de recherche en économie agroalimentaire, Université Laval.

2 Nicolas, F. et E. Valceschini (1993), «La négociation de la qualité », Économie Rurale, n² 217, sept.-oct., p. 12-17.

3 Sylvander, B. et J.C. Olivier (2002), « Avis sur la notion de qualité $»$, Avis $n^{\circ} 36$, site Web :

http://www.agriculture.gouv.fr/alim/part/

AvisNotionqualit\%E9250302.pdf

4 Idem.

5 Bonny, S. (2000), « Les consommateurs, l'agriculture, la qualité et la sécurité des aliments : une analyse du questionnement des consommateurs et des réponses apportées ", Productions Animales, INRA, n 13, p. 287-301.

6 Roger, C. (2001), « Agriculture raisonnée, multifonctionnelle, biologique...Quelle voie vers une agriculture durable?», http://www.inra.fr/Internet/Départements/ESR/ comprendre/js/agri_raison.html

7 Lacombe (1998), «Les agriculteurs et la société : quelles fonctions, quelles légitimités ?», in Miclet, Siriex, Toyer (dir.), Agriculture et alimentation en quête de nouvelles légitimités, Paris, Économica.

8 Hervieu, B. (2001), «La multifonctionnalité : un enjeu pour l'agriculture du $\mathrm{XXI}^{\mathrm{e}}$ siècle», conférence prononcée à l'Université Laval.

9 Miclet, Siriex et Toyer (1998), op. cit.

10 La réforme de la PAC en 1992, ne remet pas en cause la politique productiviste, mais prône une présence de l'agriculture, surtout du territoire. Pour la première fois apparaissent les termes d'aménagement du territoire et de préservation de l'environnement. Elle est axée autour de l'idée de multifonctionnalité et de développement rural.

11 Roger (2001), op. cit.

12 Le concept de multifonctionnalité de l'agriculture a fait son apparition au niveau international lors des négocia- tions du CNUED en 1992, puis a de nouveau été évoqué lors de l'Uruguay Round en 1994 et lors du Sommet mondial de l'alimentation en 1996. Il exprime la prise en compte de trois préoccupations majeures au sein de l'agriculture à l'heure actuelle. La première, réaffirmer le lien fondamental entre agriculture, environnement et développement durable. La seconde, renforcer les relations entre agriculture et sécurité alimentaire. La dernière, souligner dans ce nouveau contexte le lien entre agriculture et commerce international.

13 Hervieu (2001) et Lacombe (1998), op. cit.

14 Ainsi, Jacques Berthelot écrivait : «L'aggravation de la crise de la vache folle et l'épidémie de fièvre aphteuse qui met un point d'orgue à quarante ans de dérive productiviste de la politique agricole commune (PAC) et plonge éleveurs et consommateurs de l'Union Européenne dans le désarroi - fournit l'occasion de procéder à la profonde réforme que, dans leur très grande majorité, les citoyens attendent $»$.

15 Ce signe de qualité créé dans les années 1990 connaitra un essor rapide. Il est utilisé essentiellement par les entreprises de transformation ou de distribution. Il marque une certaine orientation des signes de la certification de qualité du produit à celle de son processus d'élaboration. La CCP « atteste qu'une denrée alimentaire ou qu'un produit agricole non alimentaire et non transformé est conforme à des caractéristiques spécifiques ou à des règles préalablement fixées, portant sur la fabrication, la transformation ou le conditionnement. Ces caractéristiques doivent être objectives, traçables, mesurables et significatives pour le consommateur ».

16 Chazoule, C. (2001), «Les processus d'innovation dans l'agriculture. Étude comparative à partir de deux mises en culture, les cas de la trufficulture et de l'arboriculture fruitière », thèse de doctorat, octobre, Montpellier, INRA.

17 Fontguyon (de), G. et al. (2003), «Qualité des produits alimentaires et marques de filière », Sociologie du travail, $\mathrm{n}^{\circ} 45$, p. 77-94.

18 Sauvé, L. et E. Valceschini (2003), « Agroalimentaire : la qualité au cœur des relations entre agriculteurs, industriels et distributeurs ", Économie et stratégies agricoles. 